\title{
A Venezuelan Case of Schmid-Type Metaphyseal Chondrodysplasia with a Novel Mutation in COL1OA1
}

\author{
Francisco Cammarata-Scalisi $^{\mathrm{a}} \quad$ Uta Matysiak $^{\mathrm{b}}$ Tanja Velten $^{\mathrm{b}}$ Michele Callea $^{\mathrm{c}}$ \\ Dianora Araque $^{a}$ Colin E. Willoughby ${ }^{d}$ Angela Galeotti ${ }^{c} \quad$ Andrea Avendaño $^{a}$ \\ a Unit of Medical Genetics, Department of Pediatrics, Faculty of Medicine, University of Los Andes, \\ Mérida, Venezuela; ${ }^{b}$ Center for Pediatrics and Adolescent Medicine, Medical Center, University of Freiburg, \\ Freiburg, Germany; ' Unit of Dentistry, Bambino Gesù Children's Hospital, IRCCS, Rome, Italy; \\ ${ }^{\mathrm{d}}$ Biomedical Sciences Research Institute, Ulster University, Coleraine, Northern Ireland, UK
}

\section{Established Facts}

- Schmid-type metaphyseal chondrodysplasia is an uncommon autosomal dominant skeletal dysplasia, with $50 \%$ risk of recurrence in descendants of affected individuals.

- It is caused by heterozygous mutations in the COL10A1 gene, encoding the $\mathrm{\alpha} 1(\mathrm{X})$ chains of type X collagen.

- Mutations in COL10A1 disrupt growth plate function and endochondral ossification.

\section{Novel Insights}

- The p.Leu633Thrfs*45 mutation detected in the COL10A1 gene is novel as well as the predicted effect of this mutation on protein function.

- This unreported frameshift mutation presents a similar phenotype found in other cases with the same type of mutation, including short stature, waddling gate, metaphyseal irregularities and coxa vara.

\section{Keywords}

COL10A1 mutation - Schmid-type metaphyseal chondrodysplasia

\section{Abstract}

Schmid-type metaphyseal chondrodysplasia (MIM 156500) is an uncommon autosomal dominant skeletal dysplasia caused by heterozygous mutations in the COL10A1 gene (MIM 120110) encoding the $\alpha 1(X)$ chains of type $X$ collagen. We report an 8-year-old girl with waddling gait, short stature, mild dorsal scoliosis, coxa vara, short lower limbs, bowing of the femurs, genu varum, and metaphyseal fraying and splaying, who is a carrier of a novel heterozygous 2-bp (c.1894_1895dupTA; p.Leu633Thrfs*45) duplication in exon 3 of the COL10A1 gene.

\section{KARGER}

(C) 2019 S. Karger AG, Basel

E-Mail karger@karger.com

www.karger.com/msy
Francisco Cammarata-Scalisi MD, MSc

Department of Pediatrics, University of Los Andes

Av. 16 of September, Mezzanine level

Mérida 5101 (Venezuela)

E-Mail francocammarata19@gmail.com 
Fig. 1. Pedigree of the family. Arrow indicates the proband (III:3) affected with Schmid-type metaphyseal chondrodysplasia.

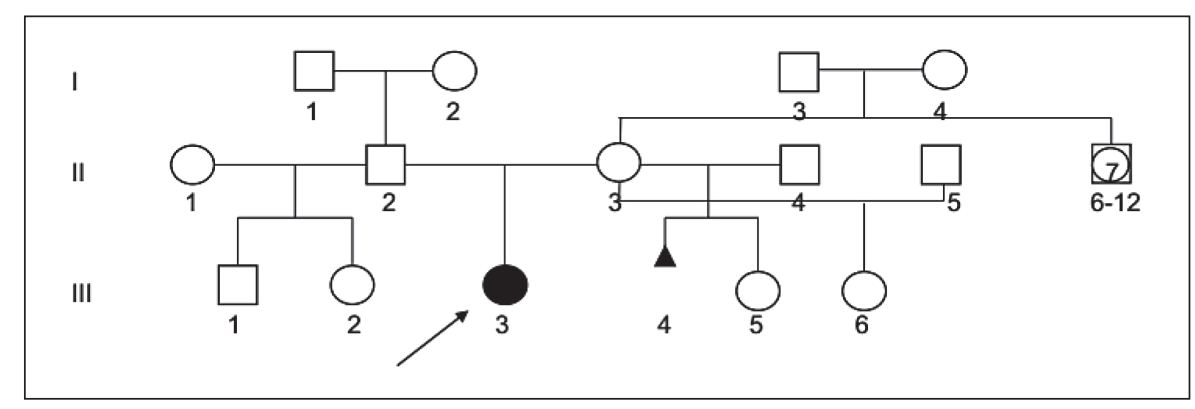

Schmid-type metaphyseal chondrodysplasia (SMCD; MIM 156500) is an uncommon autosomal dominant skeletal dysplasia, characterized clinically by mild to moderate disproportionate short stature, short legs, bowing of the long bones, and a waddling gait [Woelfle et al., 2011; Zhu et al., 2011; Park et al., 2015; Higuchi et al., 2016; Wang et al., 2017]. Radiographic findings include metaphyseal abnormalities, widening and irregularity of the growth plates, scoliosis [Park et al., 2015], shortening of tubular bones, coxa vara, and genu varum [Woelfle et al., 2011; Zhu et al., 2011; Wang et al., 2017]. The distal femoral and proximal tibial metaphyses are the most severely affected sites [Park et al., 2015], and the lumbar spine, anterior ribcage, forearms, and hands can also be affected [Woelfle et al., 2011].

SMCD is caused by heterozygous mutations in the COL10A1 gene (MIM 120110), located at 6q22, encoding the $\alpha 1(\mathrm{X})$ chains of type $\mathrm{X}$ collagen [Mäkitie et al., 2005; Woelfle et al., 2011; Higuchi et al., 2016; Wang et al., 2017]. Type $\mathrm{X}$ collagen is a short chain, non-fibrillar collagen [Park et al., 2015] which is a homotrimeric molecule consisting of 3 identical $\alpha 1(\mathrm{X})$ chains [Park et al., 2015; Higuchi et al., 2016]. Each $\alpha 1(\mathrm{X})$ chain contains a core triplehelical domain, composed of uninterrupted repeats of the Gly-Xaa-Yaa tripeptide, flanked by 2 globular domains (noncollagenous domains [NC1] and [NC2]) at both the amino and carboxyl-terminal ends [Zhu et al., 2011; Higuchi et al., 2016]. Type X collagen is an extracellular matrix protein which is normally expressed in the hypertrophic zone of the growth plate adjacent to the calcifying cartilage [Mäkitie et al., 2005; Zhu et al., 2011]. Mutations in COL10A1 disrupt the growth plate function and endochondral ossification [Woelfle et al., 2011; Park et al., 2015; Wang et al., 2017]. Histological studies showed a decreased tendency of growth plate chondrocytes to form normal cell columns [Woelfle et al., 2011]. Herein, we report the clinical and radiological findings of a patient with SMCD carrying a novel mutation in the COL10A1 gene.
Table 1. Measurements of different body segments in percentiles or standard deviation

\begin{tabular}{lll}
\hline Segments & Measurements & Percentile or SD \\
\hline Height & $111.5 \mathrm{~cm}$ & $-3 \mathrm{SD}$ to $-4 \mathrm{SD}$ \\
Weight & $19 \mathrm{~kg}$ & $3-10$ \\
Head circumference & $50.1 \mathrm{~cm}$ & $3-10$ \\
Upper arm length & $20.9 \mathrm{~cm}$ & $<3$ \\
Forearm length & $16.8 \mathrm{~cm}$ & $<3$ \\
Hand length & $14.4 \mathrm{~cm}$ & $25-50$ \\
Upper leg (thigh) length & $29.1 \mathrm{~cm}$ & $-2 \mathrm{SD}$ \\
Lower leg (calf) length & $28.5 \mathrm{~cm}$ & 10 \\
Foot length & $19.5 \mathrm{~cm}$ & $3-25$ \\
Upper segment & $57.6 \mathrm{~cm}$ & $<3$ \\
Lower segment & $53.9 \mathrm{~cm}$ & - \\
\hline
\end{tabular}

Age of the patient is 8 years and 3 months.

\section{Case Report}

The proposita was an 8-year-old Venezuelan girl, born prematurely at 33 weeks of gestation following a complicated pregnancy with oligohydramnios, preeclampsia, and severe anemia. At birth, her length was $44 \mathrm{~cm}(-1.2 \mathrm{SD})$, and weight was $1.1 \mathrm{~kg}(-4.1 \mathrm{SD})$. She was the only daughter of nonconsanguineous parents with no pre-existing family history of SMCD (Fig. 1).

She demonstrated normal psychomotor development and was referred due to short stature and skeletal dysplasia. Clinical findings included short lower limbs, genu varum, bowing of the femur and waddling gait. Measurements of the different body segments are presented in Table 1. Bone age corresponded to chronological age, and mild dorsal scoliosis, coxa vara, metaphyseal fraying and splaying were evident in radiographs of the lower limbs (Fig. 2). The cytogenetic analysis confirmed 46,XX in 40 analyzed metaphases.

\section{Methods and Results}

Genomic DNA was extracted from the patient's peripheral blood leukocytes by standard methods [Sambrook et al., 1989]. Sequencing of the COL1OA1 gene detected a novel heterozygous 2-bp duplication (c.1894_1895dupTA; p.Leu633Thrfs*45) in exon 

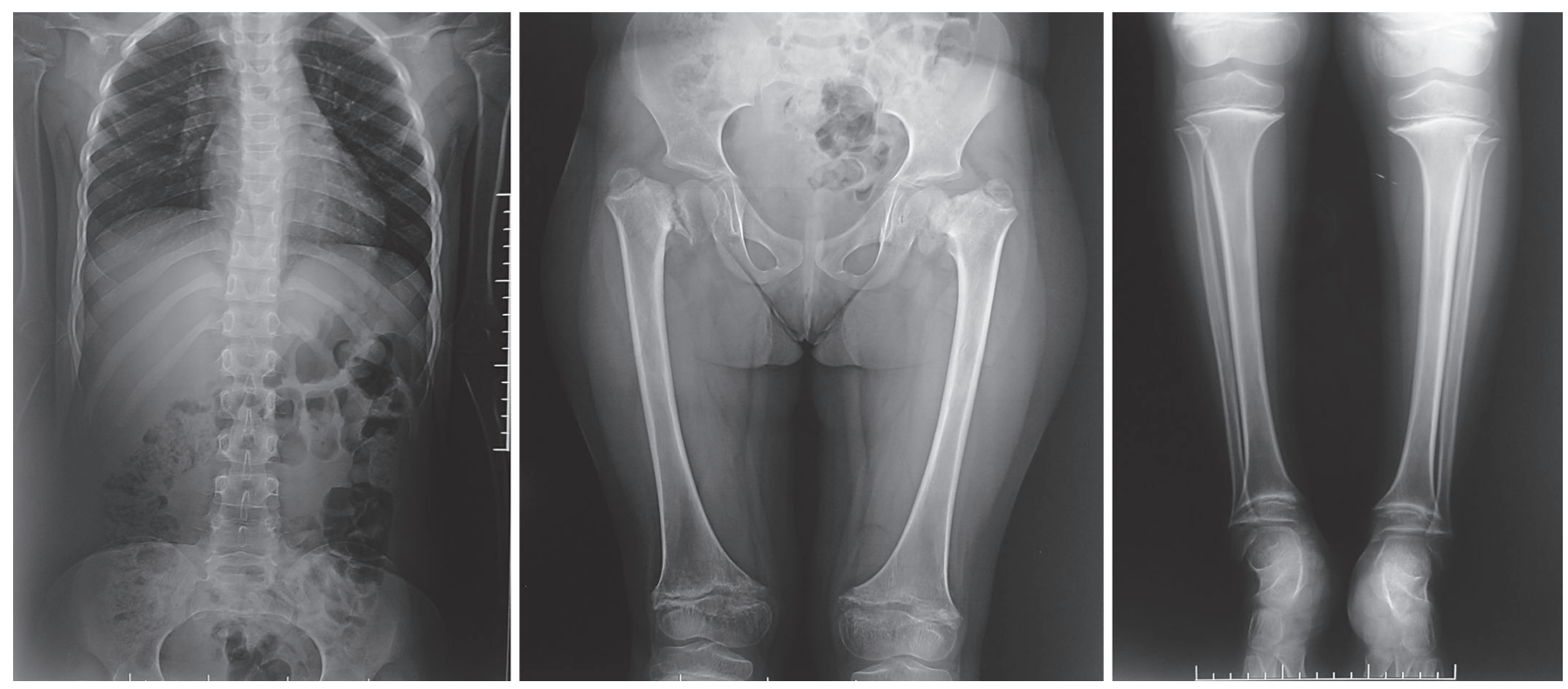

Fig. 2. Radiographs of the patient showing mild dorsal scoliosis, coxa vara, metaphyseal widening, and irregularity of the distal femoral and proximal tibia.

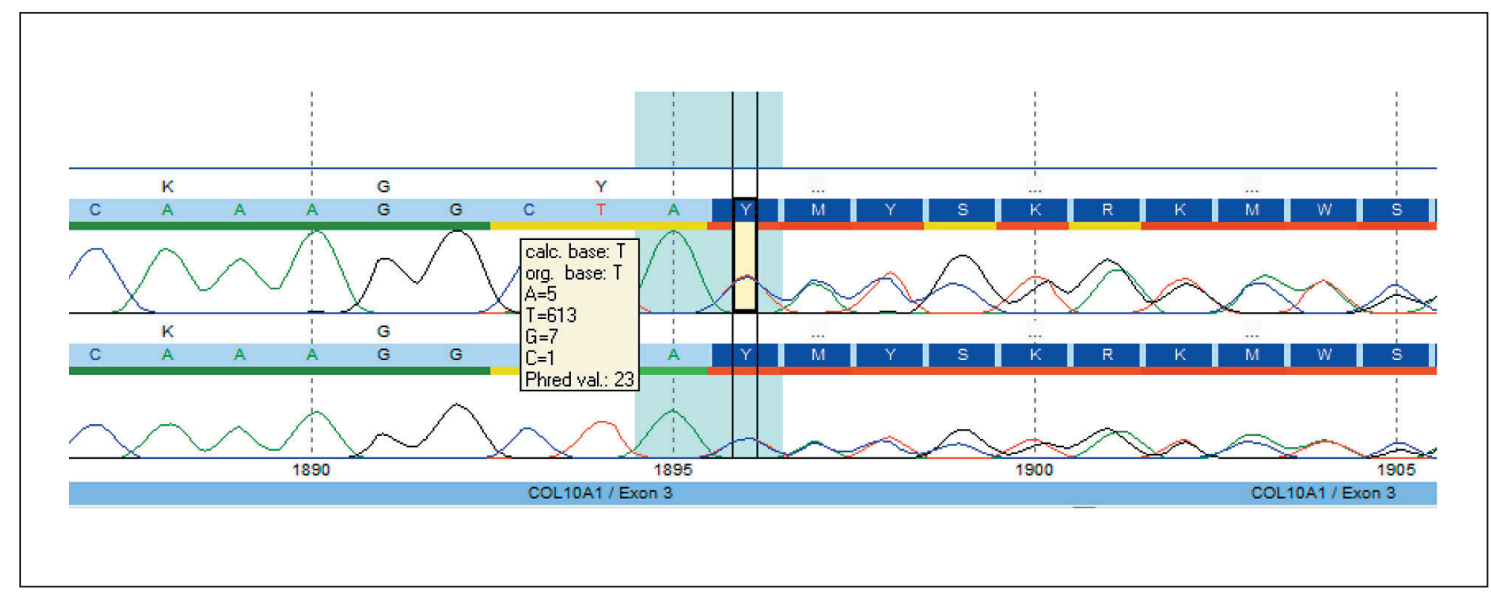

Fig.3.DNA sequenceelectropherogram of the COL10A1 gene, exon 3 of the patient showing thec.1894_1895dupTA mutation.

3 (Fig. 3), resulting in a frameshift mutation. This mutation was novel and had not been previously reported in online databases, including dbSNP, the 1000 Genomes Project, Exome Aggregation Consortium (ExAC), and the NHLBI Exome Sequencing Project. The duplication creates a frameshift starting at codon Leu633 located in the nonhelical region (NC1) of the protein resulting in 41 amino acid substitutions in C-terminal of the protein and a STOP codon 44 positions downstream of the duplication (p.Leu633Th$\mathrm{rfs}^{*} 45$ ); this is expected to produce a truncated protein of $677 \mathrm{ami}$ no acids instead of 680 amino acids.

A Venezuelan Case of SMCD

\section{Discussion}

The diagnosis of metaphyseal chondrodysplasia is clinically challenging as all long bones of the growing human skeleton can be potentially affected [Woelfle et al., 2011]. The clinical and radiological findings of SMCD are similar to those caused by rickets. Genetic analysis may be required to assist in the differential diagnosis between SMCD and rickets, especially in the cases with no biological abnormality [Higuchi et al., 2016]. 
Following the initial report of the COL10A1 gene mutation in SMCD [Mäkitie et al., 2005], at least 48 different heterozygous COL10A1 mutations have been reported in families with SMCD of different ethnic origin [Higuchi et al., 2016]. To date, the majority of mutations occur within the C-terminal NC1 domain [Mäkitie et al., 2005; Park et al., 2015; Higuchi et al., 2016; Goyal et al., 2018], which contains motifs that promote trimerization of the $\alpha 1(\mathrm{X})$ chains and subsequent formation of the triple helix to yield stable collagen X molecules [Higuchi et al., 2016; Goyal et al., 2018]. Missense, nonsense, and frameshift mutations have been reported in the $\mathrm{NC} 1$ domain, and in $53 \%$ of the cases, premature termination codons resulting from nonsense and frameshift mutations have been reported. In a murine model of SMCD, nonsense mutations lead to mRNA nonsense-mediated decay and haploinsufficiency. In SMCD, expressed mutant $\alpha 1(\mathrm{X})$ chains are unable to trimerize to form a stable collagen and accumulate intracellularly leading to endoplasmic reticulum stress in hypertrophic chondrocytes [Zhu et al., 2011].

The p.Leu633Thrfs*45 mutation detected in this study is novel and the predicted effect of this mutation on protein function was assessed using MutationTaster software [Schwarz et al., 2014], a free software that uses several algorithms and databases to estimate the impact of mutations on proteins. The amino acid substitutions in $\alpha 1(\mathrm{X})$ chains of type $\mathrm{X}$ collagen are in a highly conserved region and are predicted to result in nonsense-mediated decay. Several frameshift mutations have been reported in the COL10A1 gene and affected patients present with a similar phenotype as seen in our case, including short stature, waddling gate, metaphyseal irregularities, and coxa vara. However, the phenotype severity and others findings such as hyperlordosis showed both inter- and intrafamilial variability [Mäikitie et al., 2005; Woelfle et al., 2011; Park et al., 2015; Higuchi et al., 2016; Goyal et al., 2018].

\section{Conclusion}

This study reports the clinical and radiological findings of a case of SMCD associated with a novel, previously unreported frameshift mutation in the COL10A1 gene. The mutation is located at the carboxyl-terminal ends in the $\mathrm{NC} 1$ domain and is predicted to produce a premature stop codon resulting in a truncated protein. Further studies are required to understand genotypephenotype correlations resulting from mutations of the COL10A1 gene in SMCD.

\section{Acknowledgment}

We would like to thank Rosalia Gumina for her support with the literature review.

\section{Statement of Ethics}

Informed consent for manuscript publication and accompanying photographs was obtained from the patient and her mother. Procedures performed were in accordance with ethical standards (the Helsinki Declaration).

\section{Disclosure Statement}

The authors have no conflicts of interest to disclose.

\section{References}

Goyal M, Gupta A, Choudhary A, Bhandari A: Schmid type metaphyseal chondrodysplasia with a novel COL10A1 mutation. Indian J Pediatr, E-pub ahead of print (2018).

Higuchi S, Takagi M, Shimomura S, Nishimura G, Hasegawa Y: A Japanese familial case of Schmid metaphyseal chondrodysplasia with a novel mutation in COL10A1. Clin Pediatr Endocrinol 25:107-110 (2016).

-Mäkitie O, Susic M, Ward L, Barclay C, Glorieux FH, Cole WG: Schmid type of metaphyseal chondrodysplasia and COL10A1 mutations findings in 10 patients. Am J Med Genet A 137A:241-248 (2005).
Park H, Hong S, Cho SI, Cho TJ, Choi IH, et al: Case of mild Schmid-type metaphyseal chondrodysplasia with novel sequence variation involving an unusual mutational site of the COL10A1 gene. Eur J Med Genet 58:175-179 (2015).

-Sambrook J, Fritschi EF, Maniatis T: Molecular Cloning: A Laboratory Manual, ed 2 (Cold Spring Harbor Laboratory Press, New York 1989).

- Schwarz JM, Cooper DN, Schuelke M, Seelow D: MutationTaster2: mutation prediction for the deep-sequencing age. Nat Methods 11:361362 (2014).
Wang B, He L, Miao W, Wu G, Jiang H, et al: Identification of key genes associated with Schmid-type metaphyseal chondrodysplasia based on microarray data. Int J Mol Med 39: 1428-1436 (2017).

-Woelfle JV, Brenner RE, Zabel B, Reichel H, Nelitz M: Schmid-type metaphyseal chondrodysplasia as the result of a collagen type $\mathrm{X}$ defect due to a novel COL10A1 nonsense mutation: a case report of a novel COL10A1 mutation. J Orthop Sci 16:245-249 (2011).

Zhu Y, Li L, Zhou L, Mei H, Jin K, et al: A novel mutation leading to elongation of the deduced $\alpha 1(\mathrm{X})$ chain results in metaphyseal chondrodysplasia type Schmid. Clin Chim Acta 412:1266-1269 (2011). 